SANDIA REPORT

SAND98-0138 • UC-706

Unlimited Release

Printed January 1998
Rit $=\mathrm{D}$

CFD 231998

\title{
Uncooled Thin Film Pyroelectric IR Detector with Aerogel Thermal Isolation
}

Judith A. Ruffner, Paul G. Clem, Bruce A. Tuttle, C. Jeffrey Brinker, William L. Warren, Robert W. Schwartz, Mark V. Raymond, H. N. Al-Shareef, Denise M. Mortimer, Robert D. Nasby, Alan J. Hurd, Tammy L. Reichert, C. S. Sriram, Rimple Bhatia, Jeff A. Bullington

\section{Prepared by}

Sandia National Laboratories

Albuquerque, New Mexico 87185 and Livermore, California 94550

Sandia is a multiprogram laboratory operated by Sandia Corporation, a Lockheed Martin Company, for the United States Department of

Energy under Contract DE-AC04-94AL85000.

Approved for public release: further dissemination unlimited.

\section{Sandia National Laboratories}
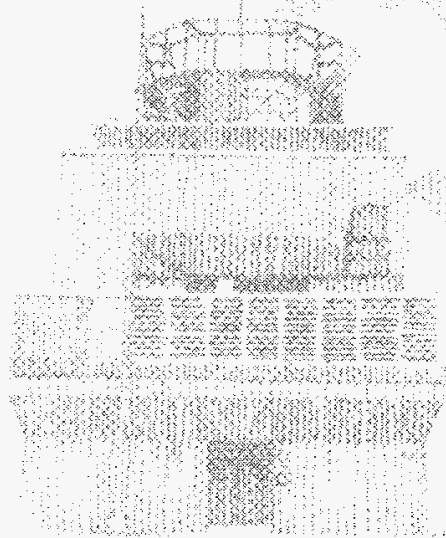

DSTPAPITON OF THIS DOCUMENT IS IMIMATIED
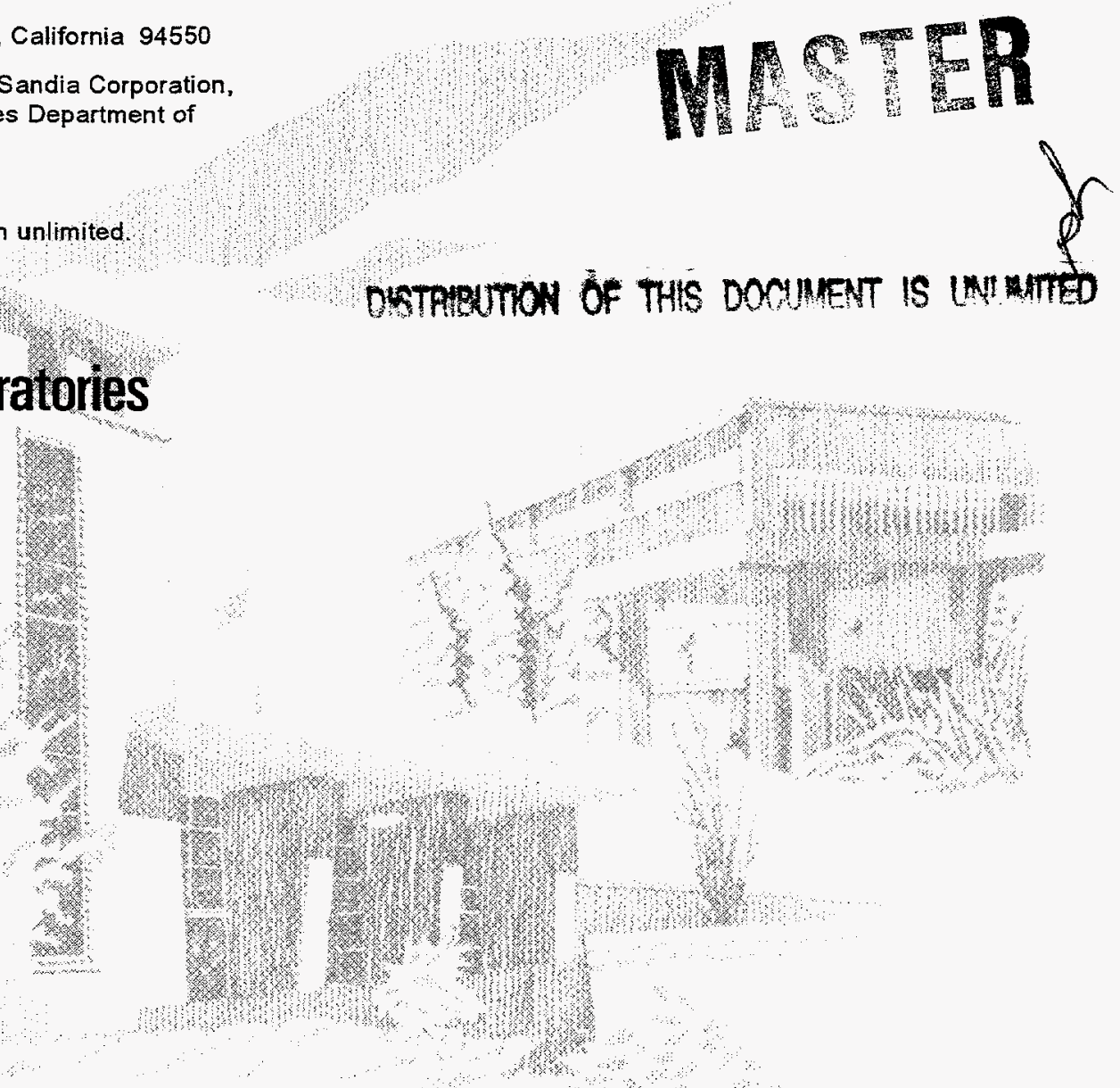
Issued by Sandia National Laboratories, operated for the United States Department of Energy by Sandia Corporation.

NOTICE: This report was prepared as an account of work sponsored by an agency of the United States Government. Neither the United States Government nor any agency thereof, nor any of their employees, nor any of their contractors, subcontractors, or their employees, makes any warranty, express or implied, or assumes any legal liability or responsibility for the accuracy, completeness, or usefulness of any information, apparatus, product, or process disclosed, or represents that its use would not infringe privately owned rights. Reference herein to any specific commercial product, process, or service by trade name, trademark, manufacturer, or otherwise, does not necessarily constitute or imply its endorsement, recommendation, or favoring by the United States Government, any agency thereof, or any of their contractors or subcontractors. The views and opinions expressed herein do not necessarily state or reflect those of the United States Government, any agency thereof, or any of their contractors.

Printed in the United States of America. This report has been reproduced directly from the best available copy.

Available to DOE and DOE contractors from

Office of Scientific and Technical Information

P.O. Box 62

Oak Ridge, TN 37831

Prices available from (615) 576-8401, FTS 626-8401

Available to the public from

National Technical Information Service

U.S. Department of Commerce

5285 Port Royal Rd

Springfield, VA 22161

NTIS price codes

Printed copy: A03

Microfiche copy: A01

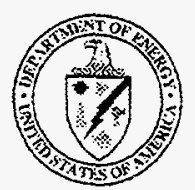




\section{DISCLAIMER}

Portions of this document may be illegible electronic image products. Images are produced from the best available original document. 
SAND98-0138

Distribution

Unlimited Release

Printed January 1998

Category UC-706

\title{
Uncooled Thin Film Pyroelectric IR Detector with Aerogel Thermal Isolation
}

\author{
Judith A. Ruffner*, Paul G. Clem*, Bruce A. Tuttle*, C. Jeffrey Brinker , William L. \\ Warren*, Robert W. Schwartz^, Mark V. Raymond*, H. N. Al-Shareef*, Denise M. \\ Mortimer*, Robert D. Nasby ${ }^{*}$, Alan J. Hurd ${ }^{+}$and Tammy L. Reichert $\downarrow$ \\ * Electronic and Optical Materials Department \\ - Direct Fabrication Department \\ ᄉ Materials Processing Department \\ * Advanced Radiation Hardened CMOS Technologies Department \\ + Theoretical and Computational Materials Modeling \\ Sandia National Laboratories \\ P. O. Box 5800 \\ Albuquerque, NM 87185-1349 \\ C. S. Sriram and Rimple Bhatia \\ Department of Chemical and Nuclear Engineering \\ University of New Mexico \\ Albuquerque, NM 87131 \\ Jeff A. Bullington \\ AMMPEC \\ Albuquerque, NM 87109
}

\begin{abstract}
Uncooled pyroelectric IR imaging systems, such as night vision goggles, offer important strategic advantages in battlefield scenarios and reconnaissance surveys. Until now, the current technology for fabricating these devices has been limited by low throughput and high cost which ultimately limit the availability of these sensor devices.

We have developed and fabricated an alternative design for pyroelectric IR imaging sensors that utilizes a multilayered thin film deposition scheme to create a monolithic thin film imaging element on an active silicon substrate for the first time. This approach combines a thin film pyroelectric imaging element with a thermally insulating $\mathrm{SiO}_{2}$ aerogel thin film to produce a new type of uncooled IR sensor that offers significantly higher thermal, spatial, and temporal resolutions at a substantially lower cost per unit.

This report describes the deposition, characterization and optimization of the aerogel thermal isolation layer and an appropriate pyroelectric imaging element. It also describes the overall integration of these components along with the appropriate planarization, etch stop, adhesion, electrode, and blacking agent thin film layers into a monolithic structure.
\end{abstract}




\section{Table of Contents}

Section

Page

Introduction 3

Experiment 5

Integrated Device Considerations $\quad 5$

Development of Aerogels 5

Planarization, Etch Stop, Adhesion, and Bottom Electrode Layers 8

Deposition and Testing of Pyroelectric Imaging Elements 9

Blacking Agent 12

$\begin{array}{ll}\text { Results and Discussion } & 12\end{array}$

Performance of Aerogels 12

Effect of Planarization Layers 13

Performance of Pyroelectric Imaging Elements $\quad 14$

Blacking Agent 18

$\begin{array}{ll}\text { Future Work } & 18\end{array}$

$\begin{array}{ll}\text { Summary } & 20\end{array}$

Appendix I - Processing Steps for a Fully Integrated, 2-Dimensional 21

Uncooled Infrared Imaging Array

References 


\section{Introduction}

Infrared detectors include both photon and thermal detectors. Photon detectors (e.g., PtSi available from Honeywell and CdTe available from Raytheon) can offer good sensitivity, fast response times, high spatial resolution, and low cost if standard semiconductor processing technology can be used. However, these detectors are sensitive to thermal noise effects and must be cryogenically cooled. The cooling units are expensive and cumbersome, making photon detectors impractical for most consumer and military applications. In addition, semiconductor infrared detectors suffer from pixel-to-pixel signal variations that make signal processing necessary in order to obtain $<1^{\circ} \mathrm{C}$ resolution. The process of mathematically normalizing the signal from each pixel reduces the signal-to-noise ratio of the array.

Thermal detectors, like pyroelectric imaging elements, offer excellent performance at room temperature and do not require cooling. They are suitable for small, light-weight detection systems that are reliable and require minimal power. Until now, these detectors have been difficult and costly to fabricate. The pyroelectric imaging elements presently manufactured by Raytheon consist of a laser reticulated barium strontium titanate (BST) ceramic that has been flat-lapped to a thickness of $<20 \mu \mathrm{m}$ and then bump-bonded to an integrated circuit. ${ }^{1}$ The lapping process is wasteful and time-consuming, and the bonding process often leads to breakage of the very delicate BST ceramic. Therefore, the technical problems with these detector elements are that throughput is low and cost is high ( $\$ 2500 /$ detector array). In addition spatial resolution is limited ( $48 \mu \mathrm{m}$ pixel size) because of the difficult bump-bonding process.

Our new monolithic device shown schematically in Figure 1 avoids the problems of the prior detectors by employing a novel structure in conjunction with standard silicon processing techniques to achieve better performance and much lower cost. ${ }^{2}$ This new IR detector is built upon a silicon substrate (which could also accommodate other microelectronic elements) on which a thermal oxide is grown as shown in the figure. Above the oxide is deposited a silica aerogel thin film to provide thermal insulation. Above this is deposited a layer of a material that serves to planarize the aerogel in order to provide a smooth and flat surface for the remaining depositions. Next is deposited an etch stop layer for later processing and a bottom electrode layer followed by the thin film pyroelectric layer. On top of the structure is the upper electrode layer capped by a blacking layer to enable the conversion of thermal radiation at the appropriate wavelengths into a heat signal $(\Delta \mathrm{T})$.

The aerogel layer in the present detector acts as an effective thermal isolation barrier between the imaging element and the relatively massive heat-sinking substrate. Effective thermal isolation enables the pyroelectric element to achieve the maximum thermal and temporal resolution for the imaging device. The use of an aerogel layer also results in greater thermal insulation and easier fabrication than the self-support "microbridge" structures shown in Figure 2 that are currently under investigation elsewhere. The use of aerogel thin films for thermal insulation may 
become the easiest, least expensive, and most reproducible fabrication method for the preparation of thin film pyroelectric arrays as a direct result of this technology.

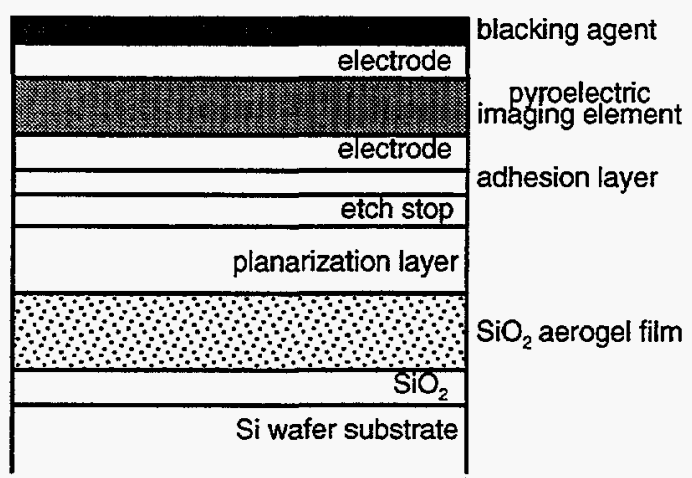

Figure 1: Schematic of monolithic uncooled infrared imaging device with silica aerogel thermal isolation layer.

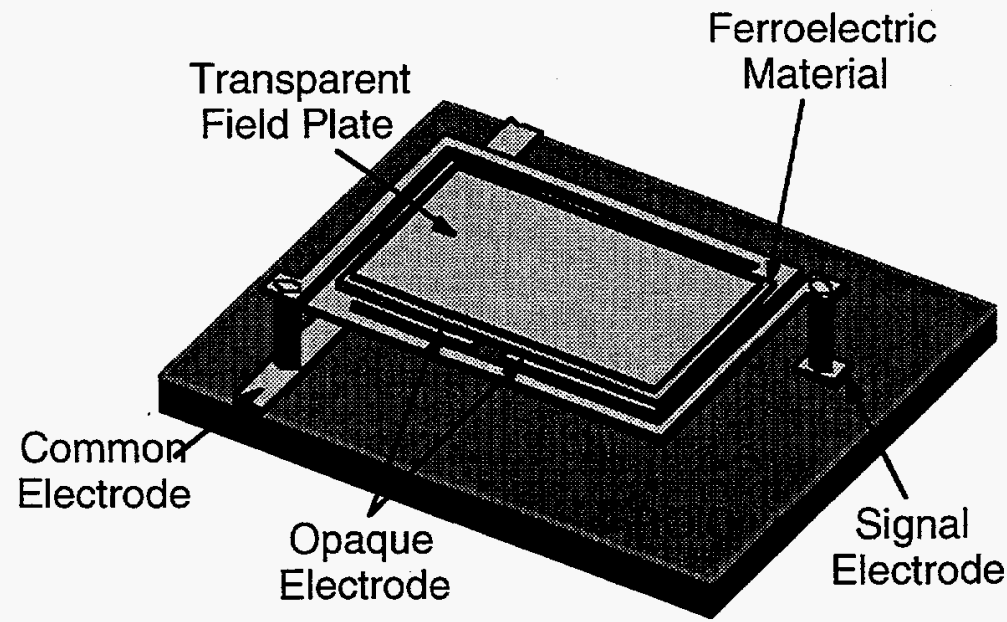

Figure 2: Schematic of an uncooled infrared pyroelectric device that uses microbridge technology to isolate thermally the imaging element from the integrated circuit substrate.

In addition, the pyroelectric imaging element of the present invention is much thinner and has a lower thermal mass than the bulk ceramic imaging elements (BST) that are presently commercially available. ${ }^{1}$ The heat capacity of the thin film imaging element is smaller, resulting in faster response times $(20-50$ times faster) and larger voltage responses. The response time is a function of thermal mass (and volume). The thickness of the total stack of the present invention is about $0.4-1.0 \mu \mathrm{m}$ as opposed to about $20 \mu \mathrm{m}$ for the Raytheon device. The noise equivalent temperature difference (NETD) of the present detector is 0.05 
${ }^{\circ} \mathrm{C}$ compared to $0.1{ }^{\circ} \mathrm{C}$ in the Raytheon product, indicating a $100 \%$ increase in the signal-to-noise ratio.

The choice of blacking agent in these devices determines their wavelength of operation. Typical wavelengths of operation are in the 3-5 $\mu \mathrm{m}$ and the 8-14 $\mu \mathrm{m}$ ranges because of high atmospheric transmission ("windows") in those spectral regions. Operation at these mid to long infrared wavelengths results in excellent sensitivity to warm objects that may not otherwise be detectable at visible or nearvisible wavelengths. For example, the peak spectral radiant exitance (emitted energy) of a human body ( $300 \mathrm{~K}$ ) occurs at approximately $10 \mu \mathrm{m}$ according to Wien's displacement law. ${ }^{3}$ As a result, a human body appears much brighter than its relatively cooler background at $10 \mu \mathrm{m}$. Image enhancers, which are photon multiplier tubes, have a wavelength of operation of $\sim 1 \mu \mathrm{m}$. While they are effective at increasing the intensity of light at that wavelength, they fail to show the strong contrast between objects at slightly different temperatures.

The fully integrated design of the present uncooled IR detector enables mass production using semiconductor processing technology. This results in excellent spatial resolution ( $4 \mu \mathrm{m}$ pixel size) at a greatly reduced cost (estimated at about $\$ 10$ $50)$.

\section{Experiment}

\section{Integrated Device Considerations}

There were a number of material and processing issues that were considered prior to design and fabrication of this monolithic structure. In particular, the porous nature of the aerogel thin film must be preserved. Because the aerogel is the first layer in the monolithic stack, all subsequent processing must be performed at temperatures that will not cause the aerogel's silica framework to densify and collapse $\left(\mathrm{T}<600^{\circ} \mathrm{C}\right)$. Next, additional solution chemistry deposition steps must be performed in such a way as to avoid filling in the aerogel pores with the new solution. This was accomplished by using an intermediate sputter deposition step to seal the top surface of the aerogel and prevent permeation of solutions used for subsequent layers into the aerogel. Finally, the aerogel surface must be fabricated or "planarized" using a second material in such a way that overlying layers are uniform in thickness.

\section{Development of Aerogels}

Recently, Brinker and co-workers demonstrated aerogel thin film deposition, whereby $0.4-2 \mu \mathrm{m}$ thickness aerogel films with up to $98 \%$ porosity may be rapidly deposited by dip coating or spin coating onto silicon wafers. ${ }^{4.5}$ This technology enables use of aerogel thin films as thermal isolation interlayers within a multilayer thin film structure. Silica-based aerogels are typically characterized by $70-99 \%$ porosity consisting of $\sim 50 \mathrm{~nm}$ interconnected pores with a $\sim 10 \mathrm{~nm}$ thick silica framework. The thermal conductivity of an aerogel decreases as porosity increases as shown in Figure 3.6 The thermal conductivity of air is plotted for comparison purposes. For porosity levels of $75-98 \%$, silica aerogels are more efficient thermal insulators (lower thermal conductivities) than air. For this application, we desire an 
aerogel film of approximately 1-2 $\mu \mathrm{m}$ thickness and a porosity level of greater than $70 \%$.

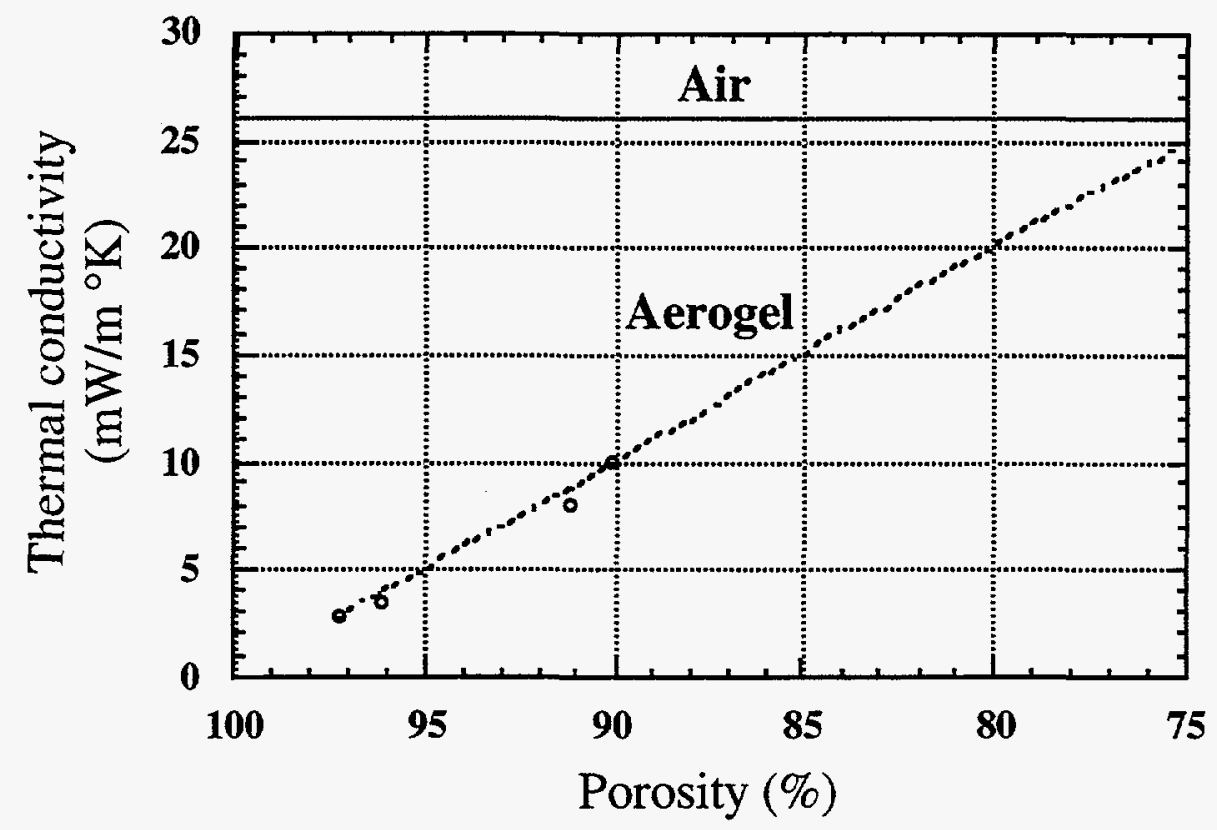

Figure 3: Thermal conductivity as a function of porosity in silica aerogels.

Silica aerogel thin films were deposited by dip coating and spin coating onto 3 and 4 inch oxidized silicon wafers, following the procedure outlined below. The resultant aerogel layer has a porosity of about $60 \%$ which is then increased to over $90 \%$ by pyrolyzing the aerogel for approximately an hour at $450^{\circ} \mathrm{C}$. The aerogel used here was based on $\mathrm{SiO}_{2}$, although a variety of other ceramics were considered as candidates. The silicon-based aerogel was chosen for this application because of its similar chemistry to the Si used for the integrated circuitry.

\section{a. Preparation of the chemical solution}

The basic starting chemicals for the preparation of the gel are the "stock solution" which is described in Table I, ethanol, and a base catalyst which is ammonium hydroxide $\left(\mathrm{NH}_{4} \mathrm{OH}\right)$ in this case.

\section{Volume}

$61 \mathrm{ml}$ Tetraethoxysilane

$61 \mathrm{ml}$ absolute ethanol

$4.87 \mathrm{ml}$ deionized water

$0.2 \mathrm{ml} 1 \mathrm{M} \mathrm{HCl}$
Approximate molar ratio

1
4
1
0.007

Table I: Components of stock solution (volumes may be multiplied) 
The chemicals were added to the reaction kettle in the order listed. Next the condenser was sealed, assembled, and heated to $60^{\circ} \mathrm{C}$ for 90 minutes with stirring. The solution was cooled and stored in a closed bottle in flammable-approved refrigerator or freezer until needed. The solution could be stored indefinitely at -20 ${ }^{\circ} \mathrm{C}$.

\section{b. Preparation of the gel}

The stock solution was allowed to warm to room temperature before use. We then added $0.05 \mathrm{M} \mathrm{NH}_{4} \mathrm{OH}$ to promote gelation which took approximately two days in an aging oven at $50^{\circ} \mathrm{C}$. The volume ratio of the stock solution : base : ethanol was $10: 1: 44$. In general, our chemical solution consisted of $10 \mathrm{ml}$ of the stock solution, $1 \mathrm{ml}$ of base and $44 \mathrm{ml}$ of ethanol. Next we aged the gel at $50^{\circ} \mathrm{C}$ in an oven for 2 more days. The gel was then ready for the pore fluid exchange washes.

\section{c. Pore fluid exchange}

The gel was washed 3 times in 3 hours with surplus ethanol (typically $\sim 60-70$ $\mathrm{ml}$ for $55 \mathrm{ml}$ of gel) at $50^{\circ} \mathrm{C}$. Then it is washed 2 times in 4 hours with surplus hexane at $50^{\circ} \mathrm{C}$.

\section{d. Surface derivatization}

Surface derivatization is the essence of the springback phenomenon which is the mechanism behind the high porosity of these films. The derivatization of the surface hydroxyl groups was performed using trimethylchlorosilane (TMCS) in hexane, typically in a volume ratio of $5: 95$. The gel was kept soaked in this surplus silylating solution for approximately 20 hours also at $50^{\circ} \mathrm{C}$.

After the derivatization was completed, the gel was washed in the final solvent that was either hexane or ethanol. If the final solvent was hexane, it was washed twice with surplus hexane in 2 hours. If the final solvent was ethanol, it the gel was washed once with surplus hexane in 1 hour followed by 2 more washes with surplus ethanol in 2 hours.

\section{e. Sonication}

Sonication involves the re-liquefication of the gel using ultrasound vibrations. As soon as the washes were completed, some additional amount of the final solvent was added to the wet gel so that the gel could be broken down ultrasonically. This resulted in a redispersed sol from which we could later deposit aerogel thin films using either spin- or dip-coating techniques.

It is important to note that the solution can be stored indefinitely at $-20^{\circ} \mathrm{C}$ after this step. It can then be warmed to room temperature and used to deposit aerogel thin films immediately, without the need for the preceding time-consuming steps each time. The two remaining steps, spin- or dip-coating and pyrolyzation, require approximately 3.0 hours to complete the deposition process. 


\section{f. Spin- and dip-coating depositions}

Oxidized silicon wafers were coated using spin- and dip-coating techniques. Pull rates ranged from 10 to 50 inches/minute while spin rates ranged from 1000 to $3000 \mathrm{rpm}$. The thickness and surface structure of the aerogel was found to be highly dependent upon the pull or spin rate. In addition, the adhesion of the aerogel film to the underlying substrate was found to be highly dependent upon the substrate cleaning procedure. Oxidized silicon wafers were cleaned in either a stream of ethanol for one minute or in an ultraviolet-ozone oven for 30 minutes prior to aerogel deposition. UV ozone ovens are used throughout the semi-conductor industry to remove organics from substrate surfaces prior to thin film deposition.

\section{g. Pyrolyzing the aerogel thin film}

As the final step in the aerogel thin film deposition, the gel is placed in a furnace in which the temperature is ramped up to $450^{\circ} \mathrm{C}$ at a rate of $10^{\circ} \mathrm{C} / \mathrm{min}$. The gel is allowed to pyrolyze for one hour at this temperature which then is ramped down at a rate of $10^{\circ} \mathrm{C} / \mathrm{min}$.

Initial attempts at deposition of the aerogel thin film using dip-coating techniques resulted in a "ribbed" pattern in which striations of variable thickness ran perpendicular to the direction of pull. In order to avoid this, we used a lower volatility solvent, heptane, instead of hexane and spin coating for all subsequent aerogel films.

The thicknesses and refractive indices of the aerogel films were measured using a Gaertner L116C ellipsometer. The volume porosity was calculated from the refractive indices using the Lorentz-Lorenz equation. Thickness values for preliminary samples were verified using a Hitachi S- 4500 field emitter scanning electron microscope, while a Nanoscope II atomic force microscope was used to characterize the surface structure of the aerogel thin films.

\section{Planarization, Etch Stop, Adhesion, and Bottom Electrode Layers}

Because of the porous nature and rough surface structure of the aerogel thin films, it was necessary to deposit a "planarization" layer on top of it. The planarization layer should be chemically compatible with the underlying silica aerogel and serve to make it planar by filling in open pores at the top surface. This layer ensures that the pyroelectric imaging element which is subsequently applied using solution chemistry deposition techniques has uniform thickness and offers a uniform response across the wafer. We chose to combine this planarization step with the deposition of the etch stop, adhesion, and bottom electrode layers.

Candidate planarization materials included $\mathrm{SiO}_{2}$ and $\mathrm{TiO}_{2}$. The $\mathrm{TiO}_{2}$ served both as a planarization layer and an etch stop layer for subsequent device processing. We then deposited a thin film of $\mathrm{Ti}$ in order to promote adhesion of the Pt layer which serves as the primary electrical conductor. A layer of $\mathrm{La}_{0.5} \mathrm{Sr}_{0.5} \mathrm{CoO}_{3}$ (LSCO) was then deposited because it has been shown to reduce the crystallization temperature of PZT sol-gel layers. Use of a lower crystallization temperature increases the survivability of the underlying aerogel layer and the entire device structure. 
These layers were sputter deposited sequentially onto silica aerogel films which ranged from 0.4 to $1.2 \mu \mathrm{m}$ in thickness using the parameters listed in Table II. Sputter deposition was performed in a UniFilm PVD-300 Multi-Source Sputter System with a base pressure of $3 \times 10^{-7}$ Torr. The aerogel thin films were heated to $250{ }^{\circ} \mathrm{C}$ in vacuum for one hour prior to deposition in order to drive off water from the porous aerogel structure and to enhance adhesion of the overlying sputtered coatings. Deposition of the planarization, etch stop, adhesion, and bottom electrode layers all took place at ambient temperature. The source-to-substrate distance was $3.6 \mathrm{~cm}$ for all materials. The $\mathrm{TiO}_{2}, \mathrm{Ti}$ and Pt layers were deposited using DC power, while the (LSCO) was deposited using RF power (13.56 MHz).

Three different thicknesses of $\mathrm{TiO}_{2}$ planarization layers were deposited in order to test the effect of thickness on the rms surface roughness. In addition, $\mathrm{TiO}_{2}$ films were deposited onto biased substrates in an attempt to planarize the top of the aerogel surface. Biasing the substrate increases the potential difference between the cathode (target) and anode (substrate). As a result, sputtered atoms arrive at the substrate with a greater kinetic energy which serves to densify the film in two ways. First, the increased energy gives the adatoms greater mobility on the substrate surface, thereby enabling them to migrate into more densely-packed, kinetically stable positions. Second, the increased kinetic energy of the incident ions results in elastic collisions with the atoms at the thin film surface. The momentum transfer pushes these atoms into voids that are near the surface of the growing film. In addition, the high incident energy causes loosely bound atoms to be ejected or "resputtered" from the film surface. These trends result in preferential thin film deposition in low spots on the substrate, such as open pores in the underlying aerogel layer.

The rms surface roughness was measured using a Nanoscope II atomic force microscope. The monolithic device up to this point included the silicon substrate, the aerogel layer, and the full electrode stack. This entire structure was annealed at $550^{\circ} \mathrm{C}$ for 30 minutes in order to crystallize the LSCO, which is typically amorphous as-sputtered.

\begin{tabular}{|c|c|c|c|c|c|c|c|}
\hline Layer & Material & $\begin{array}{c}\text { Thickness } \\
(\mathrm{nm})\end{array}$ & $\begin{array}{c}\text { Chamber } \\
\text { pres. (mTorr) }\end{array}$ & $\begin{array}{c}\text { Ar pres. } \\
(\mathrm{mTorr})\end{array}$ & $\begin{array}{c}\mathrm{O}_{2} \text { pres. } \\
(\mathrm{mTor})\end{array}$ & $\begin{array}{c}\text { Deposition } \\
\text { rate (nm/min) }\end{array}$ & $\begin{array}{c}\text { Power } \\
(\mathrm{W})\end{array}$ \\
\hline $\begin{array}{c}\text { Planarization/ } \\
\text { etch stop }\end{array}$ & $\mathrm{TiO}_{2}$ & $\begin{array}{c}100,500, \\
\text { or } 1,000\end{array}$ & 10 & 2.5 & 0.1 & 11 & $137(\mathrm{DC})$ \\
\hline Adhesion & $\mathrm{Ti}$ & 20 & 10 & 2.5 & 0 & 4.8 & 27 (DC) \\
\hline $\begin{array}{c}\text { Bottom electrode } \\
\text { layers }\end{array}$ & $\mathrm{Pt}$ & 150 & 10 & 2.5 & 0 & 6.4 & $39(\mathrm{DC})$ \\
$\mathrm{LSCO}$ & 60 & 10 & 3.3 & 0.1 & 1.6 & $64(\mathrm{RF})$ \\
\hline
\end{tabular}

Table II: Sputter deposition parameters for planarization, etch stop, adhesion, and bottom electrode layers.

\section{Deposition and Testing of Pyroelectric Imaging Elements}

Integrated ferroelectric thin films possess several attractive qualities for use in uncooled infrared (IR) pyroelectric imaging arrays. The high pyroelectric coefficients $\left[\mathrm{p}\left(\mathrm{nC} / \mathrm{cm}^{2} \mathrm{~K}\right)=\mathrm{dP} / \mathrm{dT}\right]$ and small thermal masses of these films, which may be integrated on silicon at low temperatures, enable efficient thin film arrays for 
infrared imaging applications. ${ }^{7.8}$ Critical factors for pyroelectric imaging applications include the pyroelectric current $(I)$, voltage figure of merit $\left(F_{v}\right)$, response time $(\tau)$, and noise equivalent temperature difference (NETD) of an imaging array ${ }^{910}$ and are listed in Table III. These important factors are defined in terms of the pyroelectric coefficient ( $\mathrm{p})$, area of the detector (A), change in temperature per change in time $(\Delta \mathrm{T} / \Delta \mathrm{t})$, dielectric constants of air $\left(\varepsilon_{\mathrm{o}}\right)$ and the pyroelectric material $\left(\varepsilon_{\mathrm{r}}\right)$, heat capacity $(C)$ and thermal conductance $(G)$. The NETD is defined as the thermal signal required to equal the inherent electronic noise in a detector (i.e. signal required to produce a signal-to-noise ratio of 1 ).

\begin{tabular}{ll}
\hline $\mathrm{l}=\mathrm{pA} \Delta \mathrm{T} / \Delta \mathrm{t}$ & pyroelectric current \\
$\mathrm{F}_{\mathrm{V}}=\mathrm{p} / 3.3 \varepsilon_{0} \varepsilon_{\mathrm{r}}$ & voltage figure of merit \\
$\tau=\mathrm{C} / \mathrm{G}$ & response time \\
$\mathrm{NETD} \propto \mathrm{G}^{1 / 2}$ & noise equivalent temp. difference \\
\hline
\end{tabular}

Table III: Selected pyroelectric imaging factors

For device applications, high sensitivity may be expressed as a low NETD, indicative of the approximate temperature variation due to system noise. Since NETD scales as the square root of substrate thermal conductance $G$, thin film pyroelectric pixels require thermal isolation from the device substrate.

The pyroelectric response of a material is dependent upon the change in spontaneous polarization and dielectric constant with respect to temperature $\left(\mathrm{dP}_{\mathrm{S}} / \mathrm{dT}\right.$ and $\mathrm{d} \varepsilon / \mathrm{dT}$ respectively). These quantities can be measured accurately and correlated with the compositions, thicknesses, and depositions and processing parameters of the integrated structure. This iterative process allows one to adjust the design and fabrication techniques to optimize pyroelectric response.

The pyroelectric thin film imaging element used in these devices was selected on the basis of its pyroelectric response over the operational temperature range $(\sim 0-$ $40^{\circ} \mathrm{C}$ ) of the sensor. Lead zirconate titanate (PZT: $\mathrm{Pb}_{\mathrm{x}} \mathrm{Zr}_{\mathrm{y}} \mathrm{Ti}_{1-\mathrm{y}} \mathrm{O}_{3}$ ), lead lanthanum zirconate titanate (PLZT: $\mathrm{Pb}_{\mathrm{x}} \mathrm{La}_{1-\mathrm{x}} \mathrm{Zr}_{\mathrm{y}} \mathrm{Ti}_{1-\mathrm{y}} \mathrm{O}_{3}$ ) and lead scandium tantalate (PST: $\mathrm{PbSc}_{0.5} \mathrm{Ta}_{0.5} \mathrm{O}_{3}$ ) were the materials of choice. The pyroelectric response of all three materials can be optimized by adjusting the composition, and all three can be pyrolyzed at temperatures that do not adversely affect the underlying silica aerogel thin film $\left(\mathrm{T}<650^{\circ} \mathrm{C}\right)$. PZT and PLZT compositions offer good pyroelectric responses and have been investigated extensively. ${ }^{11}$ PST is a relative newcomer to the field of pyroelectrics, but has shown excellent pyroelectric response in the temperature range of interest. ${ }^{12,13,14}$

PZT thin films were deposited by solution deposition using a methanol-based "inverted mixing order" (IMO) metal acetate/metal alkoxide system described in detail by Schwartz et al.. ${ }^{15}$ Solutions of $0.4 \mathrm{M}$ concentration were spin coated at 3000 rpm onto substrates such as platinized silicon. It was possible reproducibly to deposit $100 \mathrm{~nm}$ thick high quality PZT films. For deposition on the electroded 
aerogels, however, modifications were necessary to address film stress and electrode smoothness issues. Typically, multiple layers of $0.1 \mathrm{M}$ solutions were spin coated on the electroded aerogel substrates, with hot plate treatments to $300{ }^{\circ} \mathrm{C}$ between each layer, and crystallization at $550^{\circ} \mathrm{C}$ for 30 minutes every third layer. Films of $100-400$ $\mathrm{nm}$ thickness were produced by repeating this cycle.

PST thin films were prepared using two different deposition techniques: sputtering and solution chemistry. Previous research on reactive RF sputtering of PST thin films has indicated that perovskite-structured PST can be directly deposited at temperatures of $500-600{ }^{\circ} \mathrm{C}$, provided that the film contains excess lead..$^{13,16}$ For that reason, we used $\mathrm{PbSc}_{0.5} \mathrm{Ta}_{0.5} \mathrm{O}_{3}$ sputtering targets with 10 and $30 \%$ excess lead for these samples. PST films and the appropriate electrode layers were deposited onto silicon or sapphire substrates using the parameters listed in Table IV. All depositions were performed in a UniFilm PVD-300 sputter system which has a base pressure of $3 \times 10^{-7}$ Torr. Because of the low thermal and electrical conductivity of the PST target, care had to be taken to upramp and downramp the power to the target slowly. We used ramp rates of $5 \mathrm{~W} /$ minute. After deposition, the crystalline structures of the sputtered PST films were analyzed using x-ray diffraction (XRD).

\begin{tabular}{|c|c|c|c|c|c|c|c|c|}
\hline Layer & Material & $\begin{array}{c}\text { Thickness } \\
(\mathrm{nm})\end{array}$ & $\begin{array}{c}\text { Temp } \\
\left({ }^{\circ} \mathrm{C}\right)\end{array}$ & $\begin{array}{c}\text { Chamber } \\
\text { pres. } \\
(\mathrm{mT} \text { Torr })\end{array}$ & $\begin{array}{c}\text { Ar pres. } \\
(\mathrm{m} \text { Torr })\end{array}$ & $\begin{array}{c}\mathrm{O}_{2} \text { pres. } \\
(\mathrm{m} \text { Torr })\end{array}$ & $\begin{array}{c}\text { Dep. rate } \\
(\mathrm{nm} / \mathrm{min})\end{array}$ & $\begin{array}{c}\text { Power } \\
(\mathrm{W})\end{array}$ \\
\hline Adhesion & $\mathrm{Ti}$ & 10 & ambient & 10 & 2.5 & 0 & 4.2 & $25(\mathrm{DC})$ \\
\hline Bottom electrode & $\mathrm{Pt}$ & 300 & ambient & 10 & 2.5 & 0 & 14.0 & $14(\mathrm{DC})$ \\
\hline Pyroelectric & $\mathrm{PST}$ & 300 & 600 & 10 & 2.5 & 0.13 & 0.9 & $70(\mathrm{RF})$ \\
\hline Top electrode & $\mathrm{Pt}$ & 300 & ambient & 10 & 2.5 & 0 & 14.0 & $14(\mathrm{DC})$ \\
\hline
\end{tabular}

Table IV: Sputter deposition parameters for PST and electrode layers. PST deposition parameters are from Watton. ${ }^{16}$

PST thin films also were deposited using solution chemistry techniques. A number of solutions and preparation techniques were tried in an attempt to reduce stress and eliminate pinholes, cracking, and delamination observed on preliminary samples. The preparation technique that resulted in the most stable PST thin film was mixing $0.31 \mathrm{~g}$ tantalum ethoxide with $1 \mathrm{ml}$ of trifluoroacetic acid. The solution was stirred for 2 minutes then $3.5 \mathrm{ml}$ of methanol was added and the solution was stirred for another 5 minutes. Next $0.77 \mathrm{~g}$ of lead acetate was added to the vial and allowed to stir for 5 minutes. Finally, $0.17 \mathrm{~g}$ of scandium acetate was added to the vial and stirred and heated until dissolution occurred ( 15 minutes). At this point, the solution was clear. This solution then was used to spin coat a PST thin film onto a silicon wafer at a rate of $3000 \mathrm{rpm}$ for 30 seconds then heated on a hot plate at $300{ }^{\circ} \mathrm{C}$ for 5 minutes. The film then was pyrolyzed in a furnace at $700^{\circ} \mathrm{C}$ for 30 minutes. The heating rate for the furnace was $20^{\circ} \mathrm{C} / \mathrm{min}$ while the cooling rate was $50^{\circ} \mathrm{C} / \mathrm{min}$.

After deposition of the appropriate pyroelectric imaging element, samples were top electroded with shadow masked, sputtered Pt electrodes (100 nm thick), 
and then electrically characterized for dielectric and ferroelectric properties using a Hewlett Packard 4284A precision LCR meter and a Radiant Technologies RT-66A test unit, respectively. Pyroelectric measurements were obtained using the ByerRoundy method, using a computer-controlled Signatone hot stage for linear heating and cooling ramp rates, and a Keithley 236 source measure unit to monitor current. Samples were poled at $300 \mathrm{kV} / \mathrm{cm}$ for 30 seconds, and held 15 minutes after poling before pyroelectric measurement. Pyroelectric measurements were repeated three times on heating and cooling to avoid any current transients associated with the film poling process.

\section{Blacking Agent}

The purpose of the blacking agent is to maximize absorption of radiation within the spectral bands of interest (3-5 $\mathrm{mm}$ and 8-14 $\mathrm{mm}$ ) and subsequently to increase the signal to noise ratio of the sensor. We chose to investigate the effectiveness of $\mathrm{La}_{0.5} \mathrm{Sr}_{0.5} \mathrm{CoO}_{3}$ (LSCO 50/50) and $\mathrm{La}_{0.85} \mathrm{Sr}_{0.15} \mathrm{CoO}_{3}(\mathrm{LSCO} 85 / 15)$ as blacking agents at those wavelengths because of their anticipated high absorption and previous successful integration into this device. By using the same material for more than one purpose, we reduced the complexity of post-deposition processing.

The LSCO 50/50 and 85/15 thin films were deposited onto silicon wafers using reactive RF sputter deposition. The LSCO films were deposited to a thickness of $150 \mathrm{~nm}$ using the same parameters listed in Table II. The samples then were either annealed to 500 or $600{ }^{\circ} \mathrm{C}$, or not annealed after deposition at all. Absorption measurements for the resultant samples were performed on a Magna System 550 Spectrometer.

\section{Results and Discussion}

\section{Performance of Aerogels}

The adhesion of the silica aerogel thin films to the underlying oxidized silicon wafer was highly dependent upon the initial substrate cleaning procedure. At first, the oxidized silicon wafers were simply rinsed in a stream of ethanol for one minute prior to aerogel deposition. However, these films were observed to delaminate at the aerogel/substrate interface during a subsequent processing step (anneal of LSCO to $550^{\circ} \mathrm{C}$ ). Therefore, improving the adhesion of the aerogel to the substrate became a critical step in realizing a monolithic thin film structure.

For this application, thicknesses of $1-2 \mu \mathrm{m}$ and porosities of $>70 \%$ were desired. Scanning electron micrographs of dip-coated samples showed thicknesses of up to $3.5 \mu \mathrm{m}$. However, these samples exhibited a "ribbed" appearance caused by periodic variations in thickness that occurred during the pull process. While these films exhibited the appropriate thicknesses and porosities (80-90\%), their large-scale nonuniformities made them unsuitable for this device application. Spin-coating was used for all subsequent depositions.

Initial attempts at spin-coating also resulted in some large-scale striations across the wafer surface. The problem was found to be the high volatility of the solvent, hexane. During the spin-coating process, the hexane was volatilizing at 
different rates across the substrate due to temperature and concentration gradients. For this reason, a lower volatility solvent, heptane, was used in place of hexane for all subsequent depositions.

Using this new deposition technique, we have been able to obtain thicknesses of $\sim 0.8 \mu \mathrm{m}$ and refractive indices of 1.07 which correspond to porosities of approximately $83 \%$. The aerogel films are uniform in thickness with a maximum variation of about $10 \%$. They are completely free of visible striations. Atomic force microscopy results indicate that the standard deviation in surface height (rms roughness) is less than $10 \mathrm{~nm}$ for all of these films. A typical aerogel surface is shown in Figure 4.

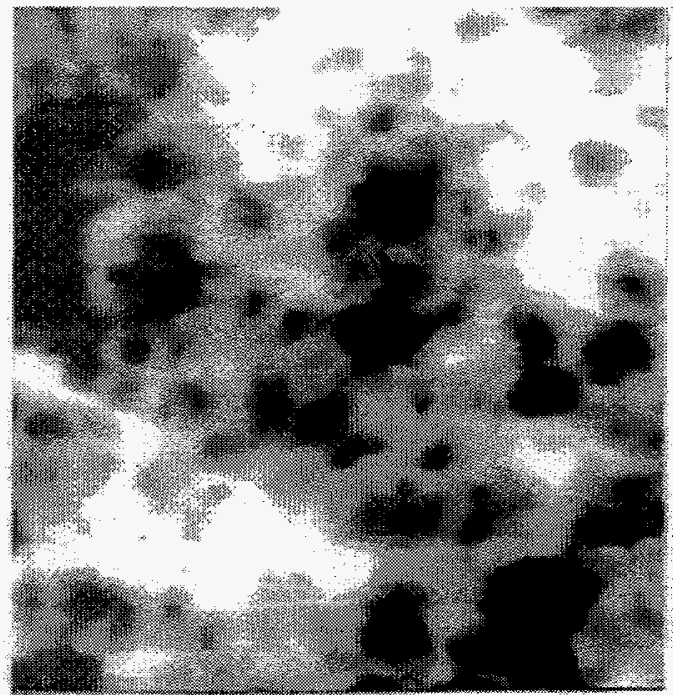

(a)

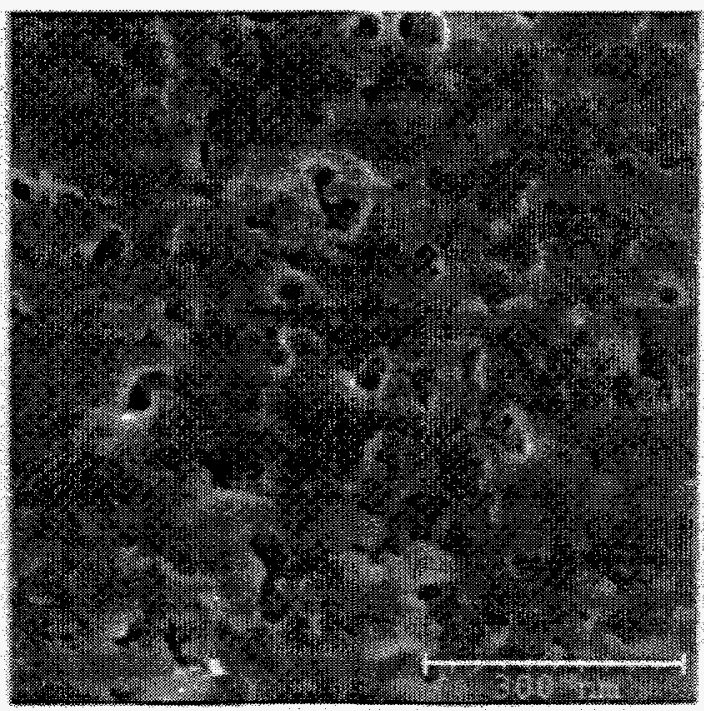

(b)

Figure 4: (a) Atomic force microscope and (b) scanning electron microscope image of a silica aerogel thin film, rms surface roughness $=8.1 \mathrm{~nm}$.

\section{Effect of Planarization Layers}

We were able to successfully sputter deposit multilayered thin film stacks directly on top of silica aerogel thin films. The sputter-deposited planarization, adhesion, and electrode layers effectively sealed the porous top surface of the aerogel and enabled subsequent solution chemistry deposition of the pyroelectric imaging element. To our knowledge, this represents the first successful integration of aerogel films with these two other deposition techniques. The resultant monolithic structures proved to be robust for processing temperatures of up to $550^{\circ} \mathrm{C}$.

$\mathrm{TiO}_{2}$ thin films of various thicknesses and processing conditions were deposited directly onto aerogel layers in order to determine their effectiveness at sealing and planarizing the aerogel surface. The resultant rms surface roughnesses for the planarization and overlying platinum and LSCO layers are summarized in Table V.

The measured rms surface roughnesses of all aerogel films were nearly equal. This was expected given that the aerogels were deposited under identical conditions, 


\begin{tabular}{|c|c|c|c|c|c|}
\hline $\begin{array}{c}\text { Planarization } \\
\text { layer } \\
(\mathrm{nm} \mathrm{TiO})\end{array}$ & $\begin{array}{c}\text { Bias voltage } \\
\text { (VDC) }\end{array}$ & $\begin{array}{c}\text { aerogel rms } \\
\text { roughness } \\
(\mathrm{nm})\end{array}$ & $\begin{array}{c}\text { Planarization } \\
\text { layer rms } \\
\text { roughness (nm) }\end{array}$ & $\begin{array}{c}\text { Pt rms } \\
\text { roughness } \\
(\mathrm{nm})\end{array}$ & $\begin{array}{c}\text { LSCO rms } \\
\text { roughness } \\
(\mathrm{nm})\end{array}$ \\
\hline 100 & 0 & 11.1 & 9.7 & 8.9 & 10.3 \\
\hline 500 & 0 & $\sim 11$ & & 8.9 & 9.4 \\
\hline 1,000 & 0 & 11.6 & 22.3 & 23.2 & 26.7 \\
\hline 1,000 & 75 & 13.7 & 9.3 & & 10.9 \\
\hline 1,000 & 150 & 11.9 & 10.3 & & 10.6 \\
\hline Table V: rms surface roughness values for various layers within multilayered \\
structure for different planarization layers.
\end{tabular}

independent of the overlying planarization layer. The surface roughness of the $\mathrm{TiO}_{2}$ planarization layer was found to be highly dependent upon its thickness. Increasing the thickness from 100 to $1,000 \mathrm{~nm}$ resulted in over twice the rms roughness (from $9.7 \mathrm{~nm}$ to $22.3 \mathrm{~nm}$ ). However, applying a bias voltage (75 VDC) to the substrate significantly reduced the roughness of the much thicker samples (from $22.3 \mathrm{~nm}$ down to $9.3 \mathrm{~nm}$ ). Increasing the bias voltage to $150 \mathrm{VDC}$ did not reduce the roughness further, suggesting that $\mathrm{TiO}_{2}$ thin films may be fully densified at lower bias voltages. The surface roughness of the uppermost LSCO layer was found to be $\sim 10 \mathrm{~nm}$ for most samples measured over a $20 \times 20 \mu \mathrm{m}$ scan area. This was sufficiently smooth for subsequent solution chemistry deposition of the pyroelectric imaging element.

\section{Performance of Pyroelectric Imaging Elements}

A composite $\mathrm{PZT} / / \mathrm{LSCO} / / \mathrm{Pt} / /$ aerogel// silicon structure was fabricated by this process. A $0.4 \mu \mathrm{m}$ thick PZT film was produced by firing at $550{ }^{\circ} \mathrm{C}$ for 30 minutes on the electroded $0.4 \mu \mathrm{m}$ aerogel film. The PZT film is characterized by a $0.3 \mu \mathrm{m}$ grain size and appears to be single phase $\mathrm{Pb}\left(\mathrm{Zr}_{0.4} \mathrm{Ti}_{0.6}\right) \mathrm{O}_{3}$, without evidence of pyrochlore or fluorite phases. The fine grain size suggests monodomain PZT grains may result, which would be advantageous if orientation can be controlled. Initial use of $0.4 \mathrm{M}$ solutions to deposit $100-400 \mathrm{~nm}$ PZT layers on the electroded aerogels gave rise to low yields and extensive shorting of PZT film capacitors. Before PZT deposition, the LSCO//Pt//aerogel//Si substrates appeared to exhibit long-range surface roughness suggested by diffuse reflection of background light. While AFM measurements indicated a relatively low surface roughness over the area studied (rms surface roughness $=10 \mathrm{~nm}$ ), a longer range roughness was suspected due to the diffuse appearance and the typical aerogel pore size $(50 \mathrm{~nm})$. Following the concept of other solution-deposition work used to planarize rough surfaces, ${ }^{17}$ multiple thin coatings of PZT were deposited by use of more dilute $0.1 \mathrm{M}$ solutions. Individual layers were heated on a hot plate to $300^{\circ} \mathrm{C}$ between depositions, and crystallized by heating to $550^{\circ} \mathrm{C}$ for 30 minutes after every $3-4$ layers. After 3-6 depositions (100 - $200 \mathrm{~nm}$ ) of these PZT thin layers, the films exhibited little specular scattering, and displayed $80-100 \%$ pixel yields. Individual heat treatment of thinner layers is also believed to minimize tensile stress 
accumulation above the aerogel, as layers undergo less densification on each heat treatment.

Ferroelectric properties of these thin films on $\mathrm{LSCO} / / \mathrm{Pt} / /$ aerogel//Si substrates were compared with identically -processed PZT layers deposited on $\mathrm{LSCO} / / \mathrm{Pt} / / \mathrm{Si}$ substrates and $\mathrm{LSCO} / / \mathrm{Pt} / / \mathrm{MgO}$ substrates to gain an understanding of substrate stress effects on ferroelectric properties. ${ }^{18,19}$ We observed that PZT films deposited on high thermal expansion coefficient oxide substrates such as $\mathrm{MgO}$ and $\mathrm{Al}_{2} \mathrm{O}_{3}$ are in compressive stress on cooling to room temperature, inducing c-axis orientation and square hysteresis loops with a large $\mathrm{P}_{\mathrm{r}}$ as shown in Figure 6. In contrast, films deposited on platinized silicon substrates are typically in tensile stress, resulting in a-axis orientation and flatter hysteresis loops. It was hoped in the current research that use of an aerogel thin film might elastically decouple the PZT film from the underlying silicon substrate, resulting in enhanced ferroelectric and pyroelectric properties. The hysteresis loop for the aerogel-coated silicon substrate shown in Figure 6 indicates an intermediate ferroelectric response. While some modest stress decoupling may be responsible for the shape of the hysteresis loop on the aerogel, the overall shape is more akin to PZT in tensile stress on silicon. Thin film $x$-ray diffraction indicated the film to be dominantly a-axis oriented atop the aerogel, further confirming this possibility. Future research will investigate whether decoupling may be achieved for individually etched $50 \mu \mathrm{m}$ pixels, rather than the coherent film on a 3 " wafer investigated here.

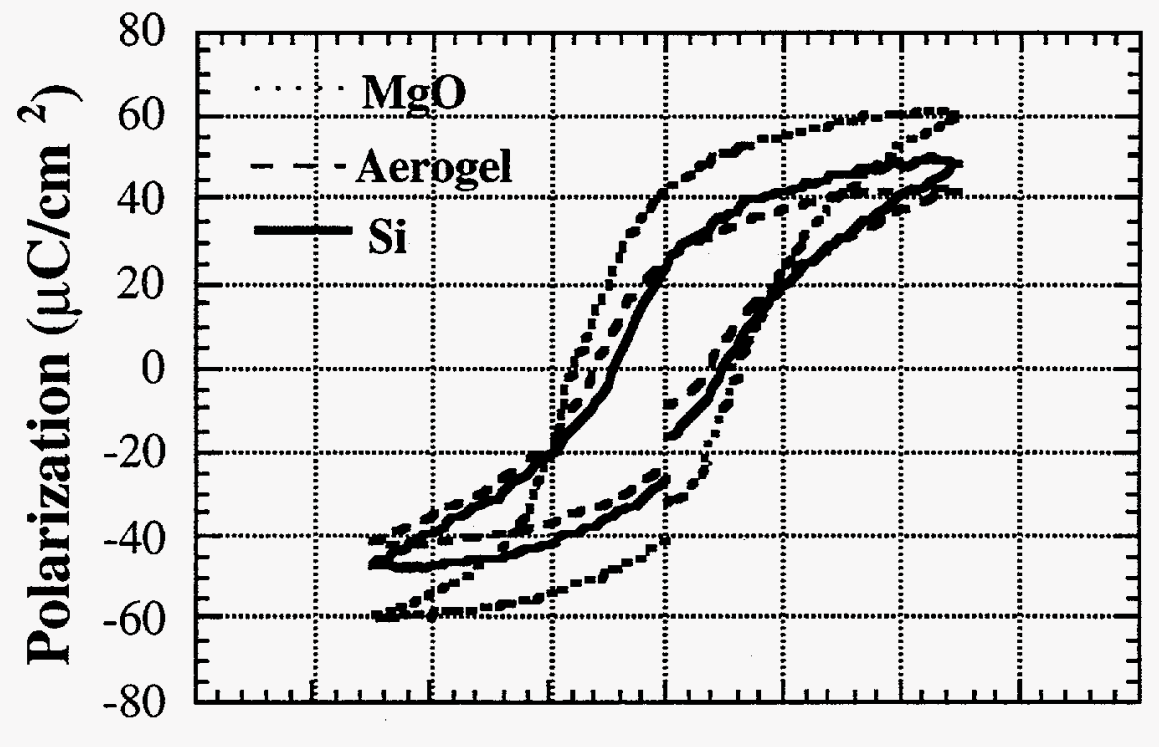

\section{Voltage (V)}

Figure 6: Hysteresis loops for $200 \mathrm{~nm}$ PZT 40/60 on LSCO//Pt//substrate for $\mathrm{MgO}$, aerogel, and Si substrates.

Pyroelectric measurements were obtained for a variety of PZT-based compositions on platinized silicon and $\mathrm{MgO}$, and for $\mathrm{Pb}\left(\mathrm{Zr}_{0.4} \mathrm{Ti}_{0.6}\right) \mathrm{O}_{3}$ on the aerogel structure. Pyroelectric measurements, voltage figures of merit, and calculated NETD values for several compositions are shown in Table VI. Films of $\mathrm{Pb}\left(\mathrm{Zr}_{0.4} \mathrm{Ti}_{0.6}\right) \mathrm{O}_{3}$ on 
the electroded aerogel displayed a pyroelectric coefficient of $30 \mathrm{nC} / \mathrm{cm}^{2} \mathrm{~K}$, and a calculated NETD of $0.07^{\circ} \mathrm{C}$, assuming $\mathrm{f} / 1.0$ optics, $\mathrm{G}=5 \times 10^{8} \mathrm{~W} / \mathrm{K}$ thermal conductance, and a $50 \mu \mathrm{m}$ pixel size. The thermal conductance value used, typical of air-gap structures, is probably conservative, since aerogels potentially offer even lower thermal conductivity. Measurements of actual thermal conductivity and conductance for aerogel structures are in progress. Several other PZT compositions appear to show promise for achieving similar NETD values. As discussed earlier, deposition of films on sapphire and $\mathrm{MgO}$ substrates has been observed to produce cdomain oriented films, with predicted greater pyroelectric coefficients as well. This appears to be the case for the highest pyroelectric coefficient observed to date, 71 $\mathrm{nC} / \mathrm{cm}^{2} \mathrm{~K}$ for PLZT $5 / 30 / 70$ on platinized $\mathrm{MgO}$, on first heating after the poling process. While results on first heating occasionally produce exaggerated pyroelectric current values, ostensibly related to post-poling phenomena, an NETD value of 0.04 ${ }^{\circ} \mathrm{C}$ was calculated for this composition. All other values in Table VI are from second and third heating cycles, with good agreement to respective values on cooling.

\begin{tabular}{|c|c|c|c|c|}
\hline Thin film & substrate & $\mathrm{p}\left(\mu \mathrm{C} / \mathrm{cm}^{2} \mathrm{~K}\right)$ & $\mathrm{F}_{\mathrm{v}}\left(\mathrm{cm}^{2} / \mathrm{C}\right)$ & NETD(calculated) \\
\hline PZT 85/15 & $\mathrm{Pt} / \mathrm{Si}$ & 0.012 & 95 & $0.17^{\circ} \mathrm{C}$ \\
\hline PZT $30 / 70$ & $\mathrm{Pt} / \mathrm{Si}$ & 0.034 & 184 & $0.09^{\circ} \mathrm{C}$ \\
\hline PLZT 4/50/50 & $\mathrm{Pt} / \mathrm{Si}$ & 0.040 & 196 & $0.08^{\circ} \mathrm{C}$ \\
\hline PLZT 3/30/70 & $\mathrm{Pt} / \mathrm{Si}$ & 0.060 & 272 & $0.06^{\circ} \mathrm{C}^{*}$ \\
\hline PTaZT $4 / 50 / 50$ & $\mathrm{Pt} / \mathrm{Si}$ & 0.064 & 313 & $0.05^{\circ} \mathrm{C}$ \\
\hline PLZT 5/30/70 & $\mathrm{LSCO} / \mathrm{Pt} / \mathrm{MgO}$ & 0.071 & 475 & $0.04^{\circ} \mathrm{C}^{*}$ \\
\hline PZT 40/60 & $\mathrm{LSCO} / \mathrm{Pt}$ /aerogel/S & 0.030 & 219 & $0.07^{\circ} \mathrm{C}$ \\
\hline
\end{tabular}

TABLE VI: Measured PZT-based thin film pyroelectric values

Pyroelectric properties were measured for several compositions of pure, lanthanum-doped, and tantalum-doped PZT, often based on PZT $30 / 70$ or $40 / 60$, since they allow low temperature $\left(550^{\circ} \mathrm{C}\right)$ crystallization. This property is expected to be advantageous for compatibility with integrated circuit metallization layers, as well as the aerogel film. The maximum aerogel processing temperature is near 600 ${ }^{\circ} \mathrm{C}$, above which the aerogel rapidly densifies, losing its thermal isolation properties. Ferroelectric properties of $\mathrm{Pb}\left(\mathrm{Zr}_{0.4} \mathrm{Ti}_{0.6}\right) \mathrm{O}_{3}$ were essentially the same for processing at either $550{ }^{\circ} \mathrm{C}$ or $600{ }^{\circ} \mathrm{C}$, with only a mild increase in $\mathrm{P}_{\mathrm{r}}$ from $28 \mu \mathrm{C} / \mathrm{cm}^{2}$ to 30.5 $\mu \mathrm{C} / \mathrm{cm}^{2}$ as shown in Figure 7 .

For some materials, $\mathrm{dP}_{\mathrm{s}} / \mathrm{dT}$ and $\mathrm{d} \varepsilon / \mathrm{dT}$ are lower in thin film than in bulk as a result of strain within films. If the pyroelectric response to changes in temperature is too low, one can enhance it by doping the films with acceptor 


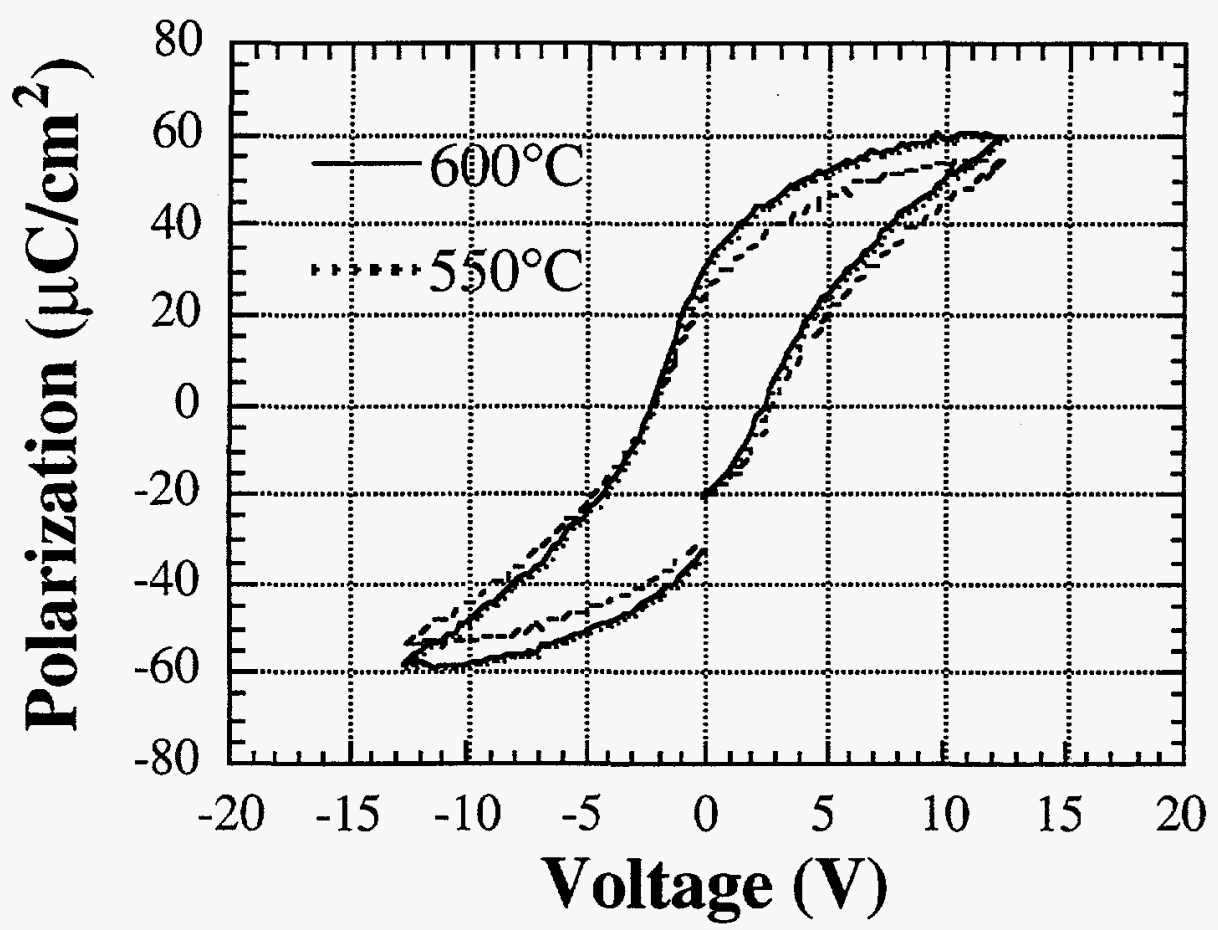

Figure 7: Hysteresis loops for $200 \mathrm{~nm}$ PZT 40/60 on LSCO//Pt//aerogel for processing temperatures of 550 and $600{ }^{\circ} \mathrm{C}$.

impurities (Fe, $\mathrm{Mn}, \mathrm{Co}, \mathrm{Ni}$ ) which are known to create charged impurity and oxygen vacancy centers. These charge centers can interact to form defect-dipoles that can be aligned to enhance polarization by biasing the dielectric. Near the Curie temperature, however, one can anticipate that the defect-dipoles become randomly oriented. This should increase the $\mathrm{dP}_{\mathrm{S}} / \mathrm{dT}$ value and increase the overall pyroelectric response significantly. In addition, we can fabricate more reliable pyroelectric films by adding dopant donors $(\mathrm{W}, \mathrm{Ta}$, and $\mathrm{Nb}$ ) that reduce imprint. Finally, one can co-dope pyroelectric films with both donors and acceptors in order to suppress the traps that cause imprint and to create defect-dipoles that enhance pyroelectric properties, respectively.

PST thin films that were fabricated using RF reactive sputter deposition and sol-gel deposition offered varying performance. PST thin films that were sputter deposited onto platinized silicon wafers were first analyzed using $x$-ray diffraction to assess their crystalline structure. The resultant $x$-ray diffraction peaks corresponded to $\mathrm{Pt}$ (111), $\mathrm{Ta}_{5} \mathrm{Si}_{2}, \mathrm{~Pb}_{2} \mathrm{ScTaO}_{6}$ (perovskite phase), and $\mathrm{Pb}_{2}\left(\mathrm{Sc}_{0.5} \mathrm{Ta}_{1.5}\right) \mathrm{O}_{6.5}$ (pyrochlore phase) in order of decreasing intensities. The presence of $\mathrm{Ta}_{5} \mathrm{Si}_{2}$ suggests that diffusion occurred through the Pt layer, enabling a chemical reaction between the $\mathrm{Ta}$ from the PST thin film and the underlying silicon substrate, probably during deposition at the elevated temperature $\left(500^{\circ} \mathrm{C}\right)$. Given the presence of this impurity, it is not surprising that the PST film failed to achieve a single phase of perovskite. The perovskite constituents were well oriented with the (220) planes parallel to the substrate as evidenced by this single diffraction peak. The PST pyrochlore phase and $\mathrm{Ta}_{5} \mathrm{Si}_{2}$ exhibited multiple diffraction peaks, indicating random orientations for those two crystalline constituents. In addition, the diffraction data 
suggests that the PST films may not be stoichiometric. The presence of $\mathrm{Ta}_{5} \mathrm{Si}_{2}$ and of $\mathrm{Pb}_{2}\left(\mathrm{Sc}_{0.5} \mathrm{Ta}_{1.5}\right) \mathrm{O}_{6.5}$ indicates an excess of tantalum in these sputtered films. The excess of Ta is likely the result of preferential sputtering of Ta compared to Sc from an otherwise stoichiometric target $(\mathrm{Sc}:$ Ta ratio $=1: 1$ ). These samples did not exhibit ferroelectric response.

Subsequent PST samples were rf reactive sputter deposited onto platinized sapphire substrates in order to prevent reactivity of the PST with the substrate material. Unfortunately, the XRD data show several diffraction peaks corresponding to $\mathrm{Ti}_{0.13} \mathrm{Ta}_{1.87} \mathrm{O}_{5}$. This indicates chemical reactivity between the $\mathrm{Ta}$ in the PST and the Ti of the adhesion layer, probably during the high temperature PST deposition. In addition, $\mathrm{XRD}$ data also revealed multiple diffraction peaks corresponding to the $\mathrm{Pb}_{2}\left(\mathrm{Sc}_{0.5} \mathrm{Ta}_{1.5}\right) \mathrm{O}_{6.5}$ pyrochlore phase, but none that corresponded to the perovskite phase. The presence of the Ta-rich pyrochlore phase in addition to the $\mathrm{Ti}_{0.13} \mathrm{Ta}_{1.87} \mathrm{O}_{5}$ compound once again indicates an excess of tantalum in these samples. More developmental work is needed for successful reactive sputter deposition of ferroelectric PST thin films.

PST thin films deposited using sol-gel techniques offered much better pyroelectric performance. The films exhibited ferroelectric behavior with a remnant polarization of $6 \mu \mathrm{C} / \mathrm{cm}^{2}$ at $10 \mathrm{kHz}$ and $100 \mathrm{mV}$ as shown in Figure 8(a). The samples offered very high "peak" pyroelectric coefficients, on the order of 100 $\mathrm{nC} / \mathrm{cm}^{2} \mathrm{~K}$, compared to the PZT and PLZT compositions. However, other data show that the pyroelectric performance of these PST thin films has not yet been optimized. For example, we anticipated a "spike" in the dielectric constant near the Curie temperature $\left(27^{\circ} \mathrm{C}\right)$ for PST. However, Figure $8(\mathrm{~b})$ shows that the dielectric constant varies as a nearly -linear function of temperature. In addition, the dielectric constant was only $\sim 740$, which is approximately an order of magnitude lower than expected. We also anticipated rapid quenching of the ferroelectric response as we tested the samples at different temperatures, away from the Curie point. However, as Figure 8(c) shows, there was significant ferroelectric activity for temperatures ranging from $5-100^{\circ} \mathrm{C}$. These trends indicate that the PST may not be

perfectly crystalline, stoichiometric, or homogeneous in composition. Nevertheless, the performance of these sol-gel deposited PST films was encouraging and optimization of the deposition process should be pursued.

\section{Blacking Agent}

The most attractive candidate for a blacking agent was the LSCO $50 / 50$ annealed to $600{ }^{\circ} \mathrm{C}$. For this material, a film of $\sim 150 \mathrm{~nm}$ in thickness displayed a transmission of only $\sim 12 \%$ in the 3-5 $\mu \mathrm{m}$ spectral range. We were unable to accurately characterize the absorption of this material in the $8-14 \mathrm{~mm}$ spectral range, but evidence and previous studies suggests that LSCO 50/50 should have reasonable absorption at these longer wavelengths as well.

\section{Future Work}

We have been successful in our attempt to fabricate a monolithic, uncooled, pyroelectric infrared detector that uses an aerogel thin film as thermal isolation. 

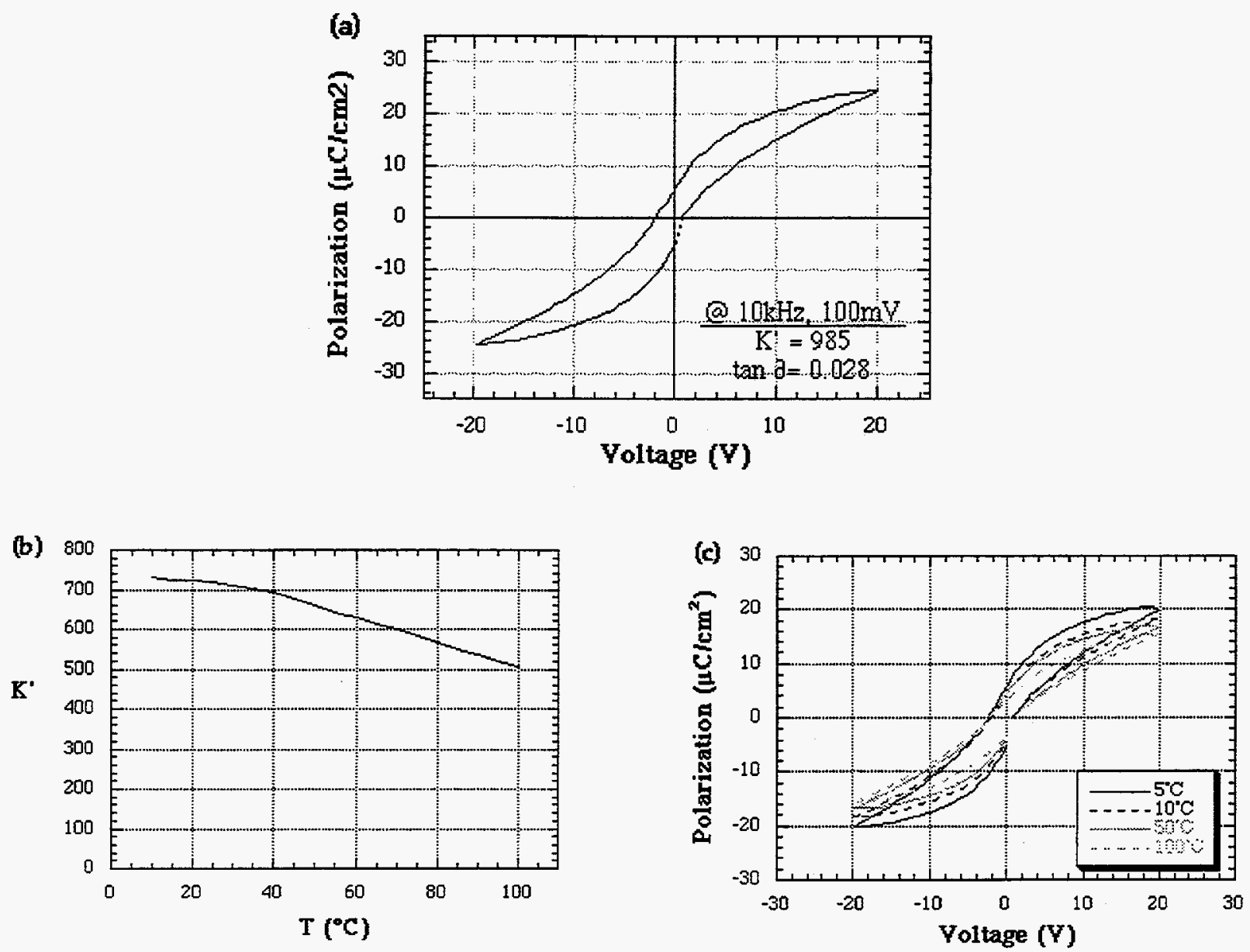

Figure 8(a) Hysteresis loop at ambient temperature (b) dielectric constant as a function of temperature and (c) hysteresis loops for several temperatures for a 350 $n m$ thick sol-gel deposited PST thin film.

The next step is to develop suitable techniques to process this monolithic structure into a 2-dimensional imaging array that is fully integrated with the appropriate read-out circuitry.

There are two main technical aspects that must be addressed: processing of the PZT or PLZT imaging array and processing of the aerogel thin film. Processing of PZT thin films has already been accomplished at Sandia National Laboratories as part of an investigation into the development of non-volatile memory devices. The general process, along with appropriate modifications required for the uncooled infrared detector application, is outlined in Appendix I.

Processing a device that contains thin films of aerogel may require some additional developmental research. The aerogels are extremely porous and consequently are susceptible to unusually high etch rates and permeation of etchants and other processing liquids into the network of pores. Dry etching techniques may be more suitable for certain processing steps. 


\section{Summary}

We have successfully fabricated several monolithic structures for uncooled infrared imaging that utilize silica aerogel thin films as thermal isolation layers. To our knowledge, this is the first reported case of successful integration of other deposition processes (sputtering and solution chemistry) with an aerogel thin film. The resultant monolithic structures showed excellent pyroelectric response with an optimum NETD value of $0.4^{\circ} \mathrm{C}$. This is less than half of the value (twice the signalto-noise ratio) offered by commercially-available uncooled infrared imaging systems at the present. In addition, the ability to fabricate this device using standard thin film deposition techniques and to process the device into a fully integrated 2dimensional imaging array using standard semiconductor techniques promises to reduce the cost of these sensors dramatically (from $\$ 2,500$ to $\$ 50$ /array).

This work was supported by Sandia National Laboratories, a multiprogram laboratory operated by Sandia Corporation, a Lockheed Martin Company, for the United States Department of Energy under contract DE-AC04-94AL85000. 


\section{APPENDIX I \\ Processing Steps for a Fully Integrated, 2-Dimensional Uncooled Infrared Imaging Array}

What follows is a listing of the process steps that can be used to produce one embodiment of the uncooled IR detector of this invention as shown in Figure 9 below. The layers are numbered corresponding to the step at which they are deposited.

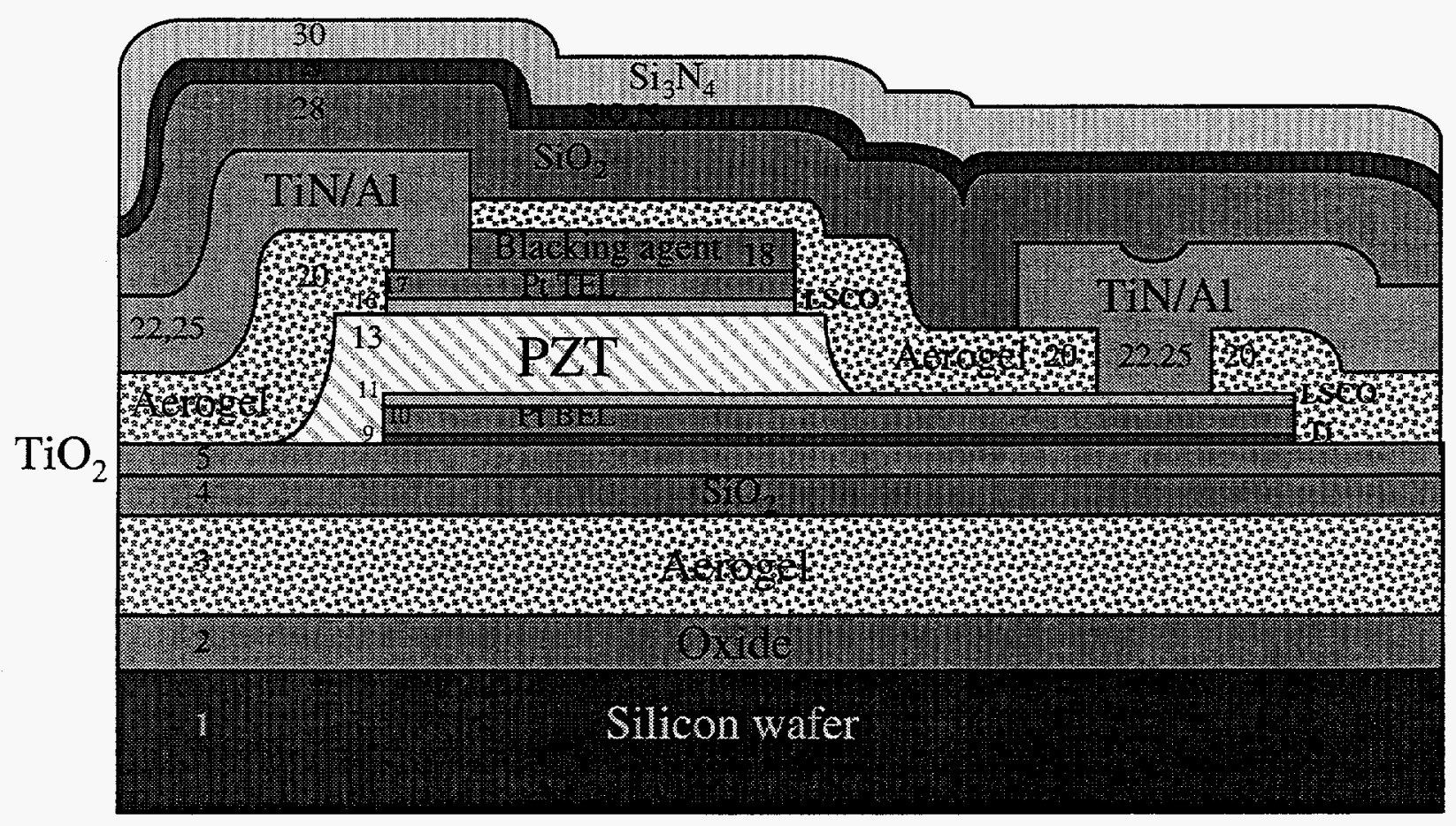

Figure 9: Schematic of fully integrated uncooled infrared detector

1. Process the silicon substrate (1) through a standard semiconductor process flow up until the process step where the first metallization is deposited. The gate of the transistor can be made from a doped polysilicon. Doped polysilicon will withstand the thermal cycling required to crystallize the ceramic/pyroelectric thin film.

2. A $1 \mu \mathrm{m}$ layer of $\mathrm{SiO}_{2}(2)$ is deposited as a protective layer on top of the underlying transistors/circuitry.

3. Deposit by spin coating an aerogel thermal insulating layer (3) on the protective dense $\mathrm{SiO}_{2}$. The aerogel is deposited by a sol-gel deposition technique and is $\sim 0.4 \mu \mathrm{m}$ thick.

4. Deposit a dense $\mathrm{SiO}_{2}$ or $\mathrm{TiO}_{2}$ layer (4) on the aerogel to encapsulate and planarize the aerogel. This can be achieved by RF sputtering. The dense layer is $\sim 20-40 \mathrm{~nm}$ thick. Alternatively, a lower porosity sol-gel could be used. 
5. On the dense $\mathrm{SiO}_{2}$ or $\mathrm{TiO}_{2}$ layer deposit a $\sim 100 \mathrm{~nm}$ of Ti metal (5) by sputter deposition or e-beam evaporation. The $\mathrm{Ti}$ will become an etch stop for a subsequent processing step. When the PZT is etched the aerogel would also be etched without the etch stop.

6. Pattern and etch Ti metal layer to yield Ti metal pads. There are several dry and wet etchants for Ti metal. One wet etchants is dilute $\mathrm{H}_{2} \mathrm{O}_{2}$.

7. Oxidize the Ti Metal to $\mathrm{TiO}_{x}$.

8. Using a lift-off technique pattern the bottom electrode stack for the

pyroelectric element. This includes a quick exposure to an $\mathrm{O}_{2}$ plasma to

remove any residual organic material for the vias.

9. Deposit a glue layer (9) for the pyroelectric metal stack. This typically is $50 \mathrm{~nm}$ of Ti metal.

10. Deposit $100 \mathrm{~nm}$ of Pt by sputtering (10).

11. Deposit $100 \mathrm{~nm}$ of LSCO (11) by sputtering Steps 8,9,10 can be executed at the same time. The Pt and LSCO together form the lower electrode.

12. Finish the patterning by removing the excess material and photoresist with an ultrasonic acetone rinse.

13. Deposit the pyroelectric ceramic thin film $\sim 1 \mu \mathrm{m}$ (13) by a sol-gel process.

14. Pattern and etch the ceramic pyroelectric material. The etchant is 6 to $1 \mathrm{BOE}+$ $3 \mathrm{HCL}+\mathrm{HNO}_{3}+10 \mathrm{H}_{2} \mathrm{O}$.

15. Using a lift-off technique, pattern the top electrode stack for the pyroelectric element. This includes a quick exposure to an $\mathrm{O}_{2}$ plasma to remove any residual organic material for the vias.

16. Deposit $100 \mathrm{~nm}$ of LSCO (16) by sputtering Steps 8, 9, 10 can be executed at the same time.

17. Deposit $100 \mathrm{~nm}$ of $\mathrm{Pt}(17)$ by sputtering. The LSCO and the Pt form the upper electrode 26.

18. Deposit the blacking element (18). Steps $16,17,18$ can be deposited at the same time.

19. Finish the patterning by removing the excess material and photoresist with an ultrasonic acetone rinse.

20. Deposit $1 \mu \mathrm{m}$ of $\mathrm{SiO}_{2}$ aerogel (20) to thermally isolate the pixels in the lateral dimensions and to serve as a protective coat.

21. Pattern and etch the $\mathrm{SiO}_{2}$ to expose the contact to the top and bottom electrode for the pyroelectric structure.

22. Deposit $50 \mathrm{~nm}$ TiN (22) metal as a barrier metal between the $\mathrm{Al}$ and pyroelectric metal contact.

23. Pattern and etch vias to the source, gate, and drain. This can be accomplished by a $\mathrm{CF}_{4}$ Plasma Etch.

24. Remove photoresist using a standard resist strip.

25. Deposit Al (25) to make contact to the pyroelectric electrode stack and semiconductor structures. (W, TiW or Cu can also be used).

26. Pattern and etch the $\mathrm{Al}$ metal.

27. Remove photoresist using a standard resist strip.

28. Deposit $\sim 2 \mu \mathrm{m} \mathrm{SiO}{ }_{2}$ layer (28) as a scratch protection. 
29. Deposit $50 \mathrm{~nm}$ of silicon oxynitride (29).

30. Deposit $200 \mathrm{~nm}$ of $\mathrm{Si}_{3} \mathrm{~N}_{4}$ (30) as an encapsulation.

31. Pattern and etch the $\mathrm{SiO}_{2}$ and $\mathrm{Si}_{3} \mathrm{~N}_{4}$ to expose bonding pads.

32. Remove photoresist using a standard resist strip.

33. Saw wafer and package. 


\section{References}

1 C. M. Hanson, K. N. Sweetser, and S. N. Frank, "Uncooled Thermal Imaging," Texas Instruments Technical Journal 11, 2-10, Sept.-Oct., 1994.

${ }^{2}$ J. A. Ruffner, J. A. Bullington, P. G. Clem, W. L. Warren, C. J. Brinker, B. A. Tuttle, and R. W. Schwartz, "Uncooled Thin Film Pyroelectric IR Detector with Aerogel Thermal Isolation," Sandia National Laboratories SD-5782, U. S. Patent Application, 1997.

3 E. L. Dereniak and D. G. Crowe, Optical Radiation Detectors, John Wiley \& Sons, New York, 1984.

${ }^{4}$ S. S. Prakash, C. J. Brinker, A. J. Hurd, and S. Rao, "Silica Aerogel Films Prepared at Ambient Pressure by Using Surface Derivatization to Induce Reversible Drying Shrinkage," Nature 374, 439-443,1995.

5 S. S. Prakash, C. J. Brinker, and A. J. Hurd, "Silica Aerogel Films at Ambient Pressure," J. Non-Cryst. Solids, 190, 264-275, 1995.

${ }^{6}$ P. Scheuerpflug, M. Hauck and J. Fricke, "Thermal Properties of Silica Aerogels between 1.4 and $300 \mathrm{~K}, "$ J. Non-Cryst. Solids 145, 196-201, 1992.

${ }^{7}$ B. A. Tuttle, P. G. Clem, J. A. Ruffner, M. A. Rodriguez, D. R. Tallant, D. Dimos, and C. J. Brinker, " $\mathrm{Pb}(\mathrm{Zr}, \mathrm{Ti}) \mathrm{O}_{3}$ Thin Film Fabrication: Process Modifications for Different Applications," Invited Talk, Workshop of the COST 514EU Action on Ferroelectric Thin Films, Parma, Italy, Apr. 15, 1997.

${ }^{8}$ B. A. Tuttle, H. N. Al-Shareef, J. A. Ruffner, C. J. Brinker, T. J. Garino, and D. Dimos, "Pb(Zr,Ti) $\mathrm{O}_{3}$ Thin Films for Integrated Sensor Applications," American Ceramic Society Conference, San Antonio, TX, Nov. 2, 1996.

9 B. M. Kulwicki, A. Amin, H. R. Beratan, and C. H. Hanson, "Pyroelectric Imaging," Proc. 8th IEEE ISAF, 1-11, 1992.

${ }^{10}$ P. W. Kruse, "Uncooled Infrared Focal Plane Arrays," Proc. 9th IEEE ISAF, 643646, 1994.

${ }^{11}$ K. K. Deb, K. W. Bennett, and P. S. Brody, "Investigation of Pyroelectric Characteristics of Lead Titanate Thin Films for Microsensor Applications," J. Vac. Sci. Technol. A 13, 1128-1132, 1995.

12 A. Patel, N. M. Shorrocks and R. W. Whatmore, "Lead Scandium Tantalate Thin Films for Thermal Detectors," in Ferroelectric Thin Films II, MRS Symposium Proc. 243, Boston, Dec. 2-4, 1991.

${ }^{13}$ S. H. Pyke, K. Z. Baba-Kishi, R. Watton, and M. A. Todd, "A Study of Reactively Sputtered Lead Scandium Tantalate Films," Integrated Ferroelectrics 4, 25-30, 1994.

${ }_{14}^{14}$ C. D. Meekison, K. Z. Baba-Kishi, R. Watton, and M. A. Todd, "Epitaxy and Interfacial Phase in Thin Films of Lead Scandium Tantalate Deposited by Reactive Sputtering on a Platinum Interlayer," Integrated Ferroelectrics 8, 283-291, 1995.

${ }_{15}$ R. W. Schwartz, R. A. Assink, and T. J. Headley, "Spectroscopic and Microstructural Characterization of Solution Chemistry Effects in PZT Thin Film Processing," in Ferroelectric Thin Films II, Mat. Res. Soc. Symp. Proc. 361, 377-387, 1995.

${ }^{16}$ R. Watton, "Ferroelectric Materials and IR Bolometer Arrays: From Hybrid Arrays towards Integration," Integrated Ferroelectrics 4, 175-186, 1994. 
17 A. S. Holmes et al., "Fabrication of Buried Channel Waveguides on Silicon Substrates Using Spin-on Glass," Applied Optics 32, 4916, 1993.

${ }_{18}$ P. G. Clem, B. A. Tuttle, J. A. Ruffner, C. J. Brinker, R. W. Schwartz, M. Rodriguez, and W. L. Warren, "Investigation of PZT//LSCO//Pt//Aerogel Thin Film

Composites for Uncooled Pyroelectric Detectors," Integrated Ferroelectrics, 1996, in press.

${ }_{19}^{19}$ P. G. Clem, B. A. Tuttle, J. A. Ruffner, C. J. Brinker, R. W. Schwartz, M. A. Rodriguez, and $\mathrm{W}$. L. Warren, "Investigation of $(\mathrm{Pb}, \mathrm{La})(\mathrm{Zr}, \mathrm{Ti}) \mathrm{O}_{3}$ Thin Film//La $0 \mathrm{Sr}_{0.5} \mathrm{CoO}_{3} / /$ Silica Aerogel Composites for Uncooled Pyroelectric IR Detectors," 9th Annual Symposium on Integrated Ferroelectrics, Santa Fe, NM, Mar. 5, 1997. 
Distribution:

Internal Distribution Only:

1 MS9018 Central Technical Files, 8940

5 MS0899 Technical Library, 4916

2 MS0619 Review and Approval Desk fo rDIE/OSTI, 12690

1 MS1436 Chuck Meyers, LDRD Office

5 MS1349 Judith A. Ruffner, 1812

1 MS1349 C.J. Brinker, 1831

1 MS1349 William Hammetter, 1846

1 MS1405 Cliff Renschler, 1812

1 MS1405 Paul Clem, 1812

1 MS1405 Bruce Tuttle, 1812

1 MS1074 Robert D. Nasby, 1306

1 MS0333 Alan J. Hurd, 1841

1 MS1411 Duane Dimos, 1831

1 MS0603 Tom Zipperian, 1313

1 MS0603 Steve Kurtz, 1312

1 MS0972 Clinton A. Boye, 5705

1 MS1415 Wil Gauster, 1112 